\title{
Validating the accuracy of activity and rumination monitor data from dairy cows housed in a pasture-based automatic milking system
}

\author{
M. F. Elischer, ${ }^{*}$ M. E. Arceo,† E. L. Karcher, ${ }^{*}$ and J. M. Siegford ${ }^{* 1}$ \\ *Department of Animal Science, Michigan State University, East Lansing 48824 \\ †Department of Animal Science, North Carolina State University, Raleigh 27695
}

\begin{abstract}
Behavioral observations are important in detecting illness, injury, and reproductive status as well as performance of normal behaviors. However, conducting live observations in extensive systems, such as pasturebased dairies, can be difficult and time consuming. Activity monitors, such as those created for use with automatic milking systems (AMS), have been developed to automatically and remotely collect individual behavioral data. Each cow wears a collar transponder for identification by the AMS, which can collect data on individual activity and rumination. The first aim of this study was to examine whether cow activity levels as reported by the AMS activity monitor (ACT) are accurate compared with live observations and previously validated pedometers [IceQube (IQ), IceRobotics, Edinburgh, UK]. The second aim of the study was to determine if the AMS rumination monitors (RUM) provide an accurate account of time spent ruminating compared with live observations. Fifteen lactating Holstein cows with pasture access were fitted with ACT, RUM, and IQ. Continuous focal observations (0600-2000 h) generated data on lying and active behaviors (standing and walking), as well as rumination. Activity recorded by live observation and IQ included walking and standing, whereas IQ steps measured cow movement (i.e., acceleration). Active behaviors were analyzed separately and in combination to ascertain exactly what behavioral components contributed to calculation of ACT "activity." Pearson correlations $\left(r_{p}\right)$ were computed between variables related to $\mathrm{ACT}$, RUM, IQ, and live observations of behavior. A linear model was used to assess significance differences in the correlation coefficients of the 4 most relevant groups of variables. Significant but moderate correlations were found between ACT and observations of walking ( $\mathrm{r}_{\mathrm{p}}$ $=0.61)$, standing $\left(\mathrm{r}_{\mathrm{p}}=0.46\right)$, lying $\left(\mathrm{r}_{\mathrm{p}}=-0.57\right)$, and activity $\left(\mathrm{r}_{\mathrm{p}}=0.52\right)$, and between ACT and IQ steps
\end{abstract}

Received March 11, 2013.

Accepted June 29, 2013

${ }^{1}$ Corresponding author: siegford@msu.edu $\left(\mathrm{r}_{\mathrm{p}}=0.75\right)$ and activity $\left(\mathrm{r}_{\mathrm{p}}=0.58\right)$ as well as between RUM and observations of rumination $\left(r_{p}=0.65\right)$. These data indicate that ACT and RUM do reflect cow walking and rumination, respectively, but not with a high degree of accuracy, and lying cannot be distinguished from standing.

Key words: behavior, sensor, data logger

\section{INTRODUCTION}

Observing animal behavior is a labor intensive and difficult task whether it is performed with live observations or though video recordings. One possible solution is the use of technology to remotely collect behavioral data on the subjects of interest. When technology is used to collect behavioral data, monitors are typically attached directly to the animal; thus, "losing sight" of the subject and therefore being unable to record data for this reason is nearly impossible. Such devices can collect data $24 \mathrm{~h} / \mathrm{d}$ over extended periods without the need to rest or download data immediately. Another advantage of such technology is that there is no observer bias related to interpreting behavior or observer effect that alters the animal's actions.

Various types of wireless sensor networks and radio frequency identification tags have been used to identify and monitor the behavior, health, and location of different livestock species such as cattle, horses, and poultry (Ruiz-Garcia et al., 2009). The most commonly used data loggers for cattle are accelerometers that record direction and speed of movement on 2 or 3 axes. When worn on the leg as a pedometer, these devices are able to record active (e.g., standing, walking) and lying behaviors (Müller and Schrader, 2003; McGowan et al., 2007; Martiskainen et al., 2009). For example, information gained from data loggers about dairy cows' activity levels (standing, walking, lying) has been examined to determine how comfortable cows are in their environments (O'Driscoll et al., 2008), where they are in the estrus cycle (McGowan et al., 2007), or what their health status is (LeBlanc, 2010). Several of the commercially available data loggers have been validated (Ledgerwood et al., 2010). 
Other types of monitors, typically utilizing microphones or vibration sensors, can provide information on cows' digestive behavior (i.e., rumination; Watanabe et al., 2008). These rumination monitors could provide insight into the cows' consummatory behaviors, which can be used as an additional method to monitor cow health and comfort (Urton et al., 2005).

However, monitoring technology can be costly in terms of money, with individual units costing up to hundreds of dollars, and in terms of time spent learning the system and generating useful data. These costs could be greatly reduced by using data loggers that are integrated with technology already being used by a farm. For example, most automatic milking systems (AMS) have sensors and software integrated into the system capable of recording various cow behaviors.

To date, there has been no research validating the accuracy of the outputs from AMS transponders with regard to either activity or rumination. Further, AMS activity monitors have been developed primarily to detect estrus and signs of illness, with software algorithms that emphasize certain types of motion (i.e., mounting) more than others. Thus, it is not known how accurate the monitors are at recording the activities they report. Therefore, the aim of this study was to validate the accuracy of outputs from AMS activity (ACT) and rumination (RUM) monitors compared with other methods of recording cow behaviors. We hypothesized that the ACT would collect information on active (e.g., standing and walking) and lying behaviors with similar reliability and accuracy to live observation and previously validated technologies. The RUM would be able to determine the difference between ingestion and rumination and accurately reflect the rumination times and patterns of each individual cow. Live observation and a previously validated data logger technology were used as the gold standards with which to compare the AMS monitor outputs.

\section{MATERIALS AND METHODS}

\section{Animals and Husbandry}

This study was conducted at the Pasture Dairy and Resource Education Center (PDREC) at the Kellogg Biological Station (KBS) in Hickory Corners, Michigan, between June 2 and July 3, 2011. Before the start of the study, all procedures were approved by the Michigan State University Institutional Animal Care and Use Committee. Housing for the lactating herd consisted of a freestall barn and pastures that were accessed via sand graze-ways. Stalls (58/pen; $1.22 \times$ $2.44 \mathrm{~m}$ ) contained waterbed mattresses that were top- dressed with wood shavings twice a week. The barn had a north-south center divide that created 2 pens of equal size, and cows remained in their assigned pen during the current lactation cycle. Each pen had 1 Lely A3 Astronaut AMS (Lely, Maassluis, the Netherlands) located at the south end of the pen. Cows only accessed the AMS associated with their pen. The cows had free access to the AMS $24 \mathrm{~h} / \mathrm{d}$. Milkings occurred on a voluntary basis, but if an individual cow had not visited the AMS for $\geq 12 \mathrm{~h}$, she was fetched by farm staff to be milked. Automated alley scrapers cleaned the barn twice a day. Stalls were cleaned and additional shavings added daily as needed.

Access to pasture was controlled via automated sorting gates (Lely) at the north end of the barn, opposite the AMS. Individual cows were identified by their transponders as they entered the sorting gate and were either allowed to exit to the pasture or were routed back into the barn if they were due for milking. Length of time since previous milking and time until next milking were automatically calculated by the AMS management program and were the factors that determined whether the individual cow was directed to pasture or back into the barn. During the study period, all lactating cows had pasture access $24 \mathrm{~h} / \mathrm{d}$ and pasture was their primary feed source. While being milked in the AMS, the cows were fed a pelleted concentrate based on their level of milk production. Additionally, all lactating cows were fed $0.91 \mathrm{~kg} / \mathrm{d}$ of coarsely ground corn distributed from a CosMix feeder (Lely).

Fifteen lactating multiparous and primiparous Holstein cows were used for this experiment (Table 1). Sixteen cows were initially enrolled; however, 1 cow was dropped post hoc due to technical problems with the AMS transponder that were discovered after completion of the experiment. All cows were at least 150 DIM, had experience with the AMS, did not require frequent fetching, and were selected across the available range of parities (overall average $2.33 \pm 0.30$ ). Two trials, each $15 \mathrm{~d}$ in length, were completed: trial $1=4$ cows $/$ AMS 1 and 3 cows/AMS 2; trial $2=4$ cows/AMS 1 and 2 . For each trial, cows were fitted with IceQube (IQ) pedometers (IceRobotics, Edinburgh, UK) on the rear pastern above the fetlock. All cows in each replication had IQ attached before data collection and removed after data collection as a group. Before entering the lactating herd, each cow was fitted with a neck collar containing an identification transponder collar using the pictorial guide provided by the manufacturer (Lely). This transponder also housed the cow activity (ACT) and rumination (RUM) monitors (Qwes-HR, Lely). Each cow was allowed a minimum of $12 \mathrm{~h}$ to habituate to wearing the devices before data collection began. 
Table 1. Characteristics of cows (15) used to validate activity (ACT) and rumination (RUM) sensors (Lely, Maassluis, the Netherlands)

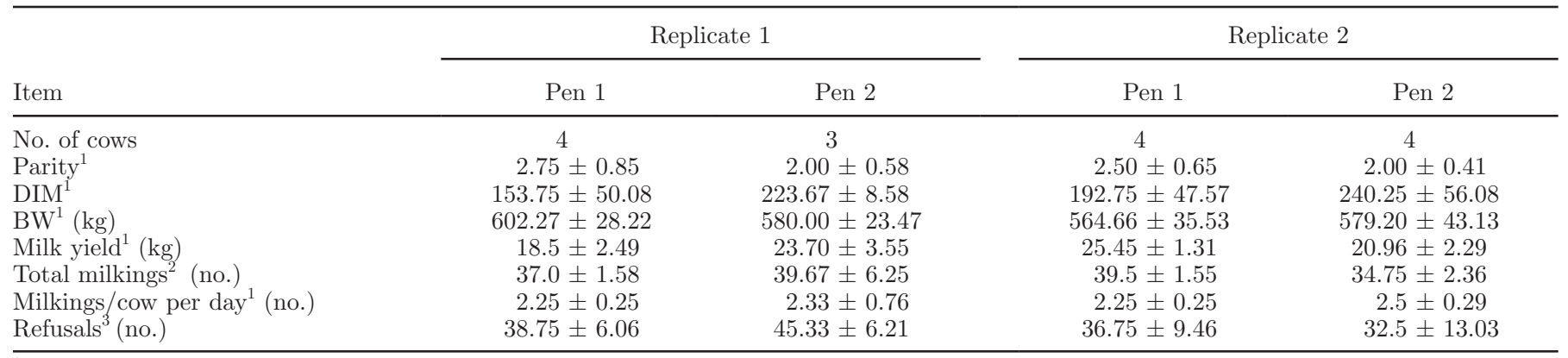

${ }^{1}$ On the first day of the replication (replicate $1=$ June 2,2011 ; replicate $2=$ June 18,2011 ).

${ }^{2}$ Total for duration of replication (replicate 1 = June 2-June 18, 2011; replicate 2: June 18-July 3, 2011).

${ }^{3}$ Refusals occurred when cows entered the automatic milking system (AMS) before their minimum milking interval had elapsed. During refusals, the AMS would immediately open without feeding the cow or attempting to milk her.

\section{Data Collection}

Four observers $(2 /$ d) were trained to perform live observations of behavior (OB) during 1 training session. Each individual was familiarized with the ethogram (Table 2) created for the project and inter-observer reliability was formally assessed between trials. To assess inter-observer reliability, all observers performed live observations on one focal cow simultaneously for the same 1-h period. Agreement between the observers was assessed using the Pearson correlation coefficient, and reliability across the 4 observers was at least $99.8 \%$.

One focal cow was watched per day for a continuous 14-h period (0600-2000 h). To ensure that observers did not become fatigued, observers were alternated every 2 $\mathrm{h}$ throughout the day with only one person observing in any given time block. Stopwatches were used to record durations of behaviors during each observation block. Continuous observations were performed to collect data on 3 distinct postural behaviors (walking, standing, and lying) and 1 digestive behavior (rumination). Each posture was mutually exclusive, but rumination could occur with any posture. Location of the cow (barn, including the AMS, graze-way, or pasture) was recorded simultaneously with postural and digestive behaviors. Binoculars were used to minimize behavioral disturbances when observing and to aid in positively identifying the focal cow. Cows were identified using a combination of ear tag number, GPS collar number, color of the GPS collar, and each cow's natural unique coloration and spot pattern.

If more than 12 min of observational data from any 2-h time block were missing, that block was not used and observations for a replacement 2-h block were collected at a later date. Twelve minutes was chosen as the maximum amount of acceptable missed time to ensure that data were collected from over $90 \%$ of each 2 -h time block.

All data from IQ, ACT, and RUM were collected and stored by the respective devices and later downloaded to a computer. The AMS monitor collected data for $\mathrm{ACT}$ and RUM in 2-h blocks in both cases. The ACT data were reported in "activity units." According to the AMS manufacturer, ACT recorded how many times the cow moved by measuring acceleration associated with upward movement of the cow's head, such as when it bobs during walking or when a cow rears up to mount another cow (Ben Smink, Lely USA Inc., Madison, WI, personal communication). Behaviors with downward head motions such as grazing or eating at the feed rail or other activities with only horizontal acceleration were not recorded (Ben Smink, personal communication). The count restarted at the beginning of every 2-h block (e.g., 0600 h, 0800 h, and so on) and when

Table 2. Ethogram used for live observations of cow behavior

\begin{tabular}{ll}
\hline Behavior & Definition \\
\hline Walk & Three or more consecutive steps made by the cow. \\
Stand & $\begin{array}{l}\text { Body is upright, supported by at least } 3 \text { legs. Standing time officially begins when the leg bearing the pedometer is fully } \\
\text { perpendicular (vertical) to the ground. }\end{array}$ \\
Lie & $\begin{array}{l}\text { Body is not supported by the legs; body is in contact with the ground. Cow is resting sternally or laterally. Lying time } \\
\text { officilly begins when the leg bearing the pedometer is parallel (horizontal) to the ground. }\end{array}$ \\
Ruminate & $\begin{array}{l}\text { Cow regurgitates bolus and chews cud. Regurgitation is demonstrated by neck and head movement and slight opening of the } \\
\text { mouth. Chewing is the cow actively moving her jaw to masticate the bolus. Bout ends when there has been no chewing or } \\
\text { regurgitation for } \geq 10 \mathrm{~s} \text { or the cow engages in another oral behavior (e.g., grazing/eating, drinking, grooming). }\end{array}$ \\
\hline
\end{tabular}


the individual was identified by an element of the AMS system (i.e., AMS, barn exit gates, or the external automatic feeder) in the barn (Ben Smink, personal communication). Regardless of when the data were downloaded, the time stamp on the data allowed the software to identify data to avoid replication. In cases where partial 2-h blocks were downloaded, the software linked counts in sequential order to provide a single summed output for each 2-h block. Higher numerical values of activity units were associated with greater activity from the cow. In this study, activity units reported for a 2-h block ranged from 9 (low activity) to 101 (high activity). Data were collected and stored by Time for Cows (T4C; Lely), the management program associated with the AMS.

The IQ data were downloaded via the IceReader and collected via IceManager (both from IceRobotics). The data were presented in spreadsheets that were rearranged as necessary for analysis. Outputs of IQ were reported in 15-min time blocks with 2 categories of posture recorded: standing (IQA) and lying (IQL). Thus, for any given 15-min interval, the total time a cow spent standing or lying was provided. The IQ also reported the number of steps as a total count from the leg with the pedometer for each 15-min time block (IQST).

For OB, the duration of a behavior was recorded continuously in real time by trained observers. Observers had stopwatches and data sheets to time and record all behaviors of interest. Activity initially included the discrete categories of walking (OBW), standing (OBS), and lying $(\mathbf{O B L})$; data from OBW and OBS were also combined into an "active" category (OBA) for better comparison with ACT and IQ data.

The output for the RUM monitors was reported in minutes per 2-hour period. The AMS transponders contain a microphone that records the sound of the bolus as it travels through the cow's throat during rumination bouts. The RUM counts boluses, chews per bolus, total rumination time $(\mathrm{min})$, and the longest rumination bout (min). In this study, total rumination time was used. Live observations of rumination (OBRUM) were recorded at the same time as the activity observations and under the same conditions (Table 2).

\section{Statistical Analysis}

All statistics were performed using SAS version 9.2 (SAS Institute Inc., Cary, NC). Statistical significance was declared at $P<0.05$. Because of differences in how each piece of technology reported activity outputs, raw data from $\mathrm{OB}$ and IQ (initially reported in minutes) were converted to percentages of time for each 2-h block to allow for comparison on the same scale with ACT activity units (9-101 activity units versus $0-100 \%$ of time). Pearson correlations (PROC CORR) were calculated to determine the strength of the relationship between the different technologies and OB.

Dependence between variables was assessed in 2 steps. First, overall correlations (Table 3) were computed for the 28 possible comparisons (all possible comparisons for the 8 variables included in this study: ACT, OBA, OBW, OBS, OBL, IQA, IQST, and IQL). Second, correlations between variables were estimated within cow, using the data on the 7 different 2-h blocks of time. The total number of observations per variable was 105 (15 cows $\times 7$ blocks of time). This last set of correlations was used to assess significant differences in 6 contrasts of interest. To define the contrasts, emphasis was placed on the 4 most relevant sets of correlations: (1) OBA with ACT, (2) OBW with ACT, (3) IQA with ACT, and (4) IQST with ACT. A linear fixed model was fitted to these data, such that

$$
y_{i j}=\mu+\operatorname{cow}_{i}+\operatorname{group}_{j}+e_{i j},
$$

where $y_{i j}$ corresponded to the correlation coefficient of cow $i$ for group $j ; \mu$ is the overall mean, cow $w_{j}$ corresponded to the effect of the $i$ th cow, with $i=1, \ldots$,

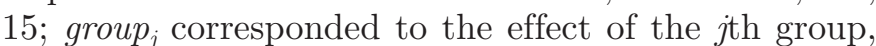
with $j=\mathrm{OBA}$ with ACT, OBW with ACT, IQA with ACT, and IQST with ACT. Pairwise significant mean differences (Tukey-adjusted $P$-values) between groups were assessed (Table 4). Finally, variables were transformed to standardize them and bring all measures into the same scale (e.g., mean $=0$, variance $=1$ ) on a cow-specific basis for graphical comparison purposes (Figure 1).

Pearson correlations were also performed on RUM and OBRUM (Table 5). Additionally, a Bland-Altman analysis and corresponding plot were generated for rumination data to determine bias and agreement between RUM and OBRUM.

After the correlations were performed on the reduced data set, 3 individual cows had RUM data with a very poor correlation with observational data, possibly indicating a malfunction of RUM. Furthermore, a linear fixed model that considered pen and trial as classificatory variables was fit to this data and formal tests were completed to determine if these cows were outliers. Two of the cows (\#2717 and \#2723) were outliers and 1 of them (\#2717) was influential according to Cook's distance (Cook's D >0.5). These cows, however, remained in the analysis as there were no known errors occurring during data collection; all rumination data are depicted in Figure 4. 
Table 3. Pearson correlations ( $P$-values in parentheses) of unstandardized data between the different technologies used to assess cow behavior ${ }^{1}$

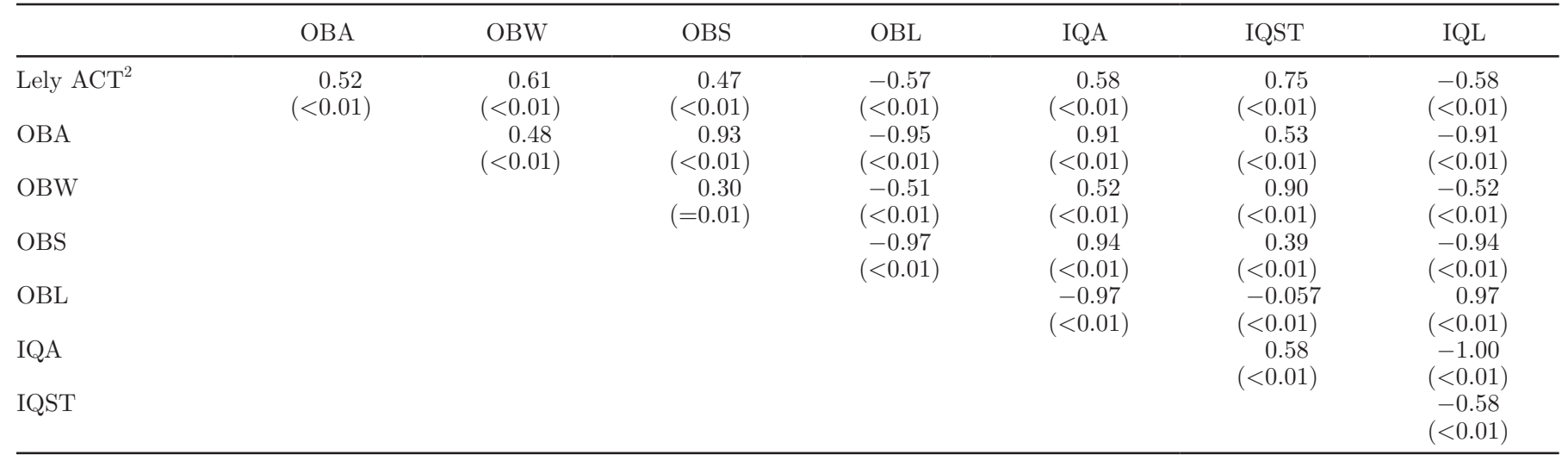

${ }^{1} \mathrm{OBA}=$ live observation active (includes standing and walking behaviors); OBW = live observation walking; OBS = live observation standing; $\mathrm{OBL}=$ live observation lying; IQA = IceQube (IceRobotics, Edinburgh, UK) active (includes both walking and standing behaviors); IQST = IceQube count of the number of steps; IQL = IceQube lying.

${ }^{2}$ Lely activity monitor (Lely, Maassluis, the Netherlands).

\section{RESULTS}

\section{Activity Data}

Moderate to strong correlations were observed between live observations (OB) and IQ pedometers in all categories of behaviors using the original, unstandardized data: active, walking, standing, and lying (Table $3)$. The strongest Pearson correlations $\left(\mathbf{r}_{\mathbf{p}}\right)$ using unstandardized data were between OBL and IQL $\left(\mathrm{r}_{\mathrm{p}}=\right.$ $0.97, P<0.0001)$, and between OBL and IQA $\left(\mathrm{r}_{\mathrm{p}}=\right.$ $-0.97, P<0.0001)$. A strong relationship between original, unstandardized data was also observed between IQST and OBW $\left(\mathrm{r}_{\mathrm{p}}=0.90, P<0.0001\right.$; Figure 2$)$. The strongest relationship found for AMS activity monitor data was that observed between ACT and IQST $\left(\mathrm{r}_{\mathrm{p}}=\right.$ $0.75, P<0.0001$; Figure 3), followed by ACT relative to OBW ( $\mathrm{r}_{\mathrm{p}}=0.61, P<0.0001 ;$ Figure 4$)$. Other ACT correlations with pedometer data and observations of active behaviors were much weaker (Table 3 ).

Significance of the 6 contrasts evaluated is shown in Table 4 . The results here indicated no significant dif- ferences (Tukey-adjusted $P$-value $<0.05)$ in the mean correlation of ACT with OBA $(\mathrm{r}=0.49)$ or with IQA $(\mathrm{r}=0.54)$. Similarly, we found no significant differences in the mean correlation of ACT with OBW $(\mathrm{r}=0.74)$ or with IQST $(\mathrm{r}=0.84)$.

\section{Rumination Data}

Upon review of the data set, we found periods when individual cows' heads were not visible to the human observer and were clearly marked as not visible on the data sheets. Because of the lack of conclusive information during these periods as to whether the individual cow was ruminating or not, these time blocks were removed from the data set. This reduced the total number of data points from 105 to 63 . The Pearson correlation between RUM and OBRUM for the periods when the cows' heads were visible was $\mathrm{r}_{\mathrm{p}}=0.65(P$ $<0.0001)$. No difference was observed between pens or trials. Correlations between RUM and OBRUM for individual cows are reported in Table 5 , and data were

Table 4. Differences of group least squares means (Tukey adjusted for multiple comparisons)

\begin{tabular}{lccc}
\hline Contrast $^{1}$ & Estimate & SE & $\begin{array}{c}\text { Adjusted } \\
P \text {-value }\end{array}$ \\
\hline OBA with ACT vs. OBW with ACT & -0.25 & 0.09 & $0.032^{\mathrm{a}}$ \\
OBA with ACT vs. IQA with ACT & -0.06 & 0.09 & 0.92 \\
OBA with ACT vs. IQST with ACT & -0.35 & 0.09 & $0.00^{\mathrm{a}}$ \\
OBW with ACT vs. IQA with ACT & 0.20 & 0.09 & 0.13 \\
OBW with ACT vs. IQST with ACT & -0.10 & 0.09 & 0.68 \\
IQA with ACT vs. IQST with ACT & -0.29 & 0.09 & $0.01^{\mathrm{a}}$ \\
\hline
\end{tabular}

${ }^{a}$ Significant difference (Tukey-adjusted $P<0.05$ ).

${ }^{1} \mathrm{OBA}=$ live observation active (includes both standing and walking behaviors); $\mathrm{ACT}=$ Lely activity monitor (Lely, Maassluis, the Netherlands); OBW = live observation walking; IQA = IceQube (IceRobotics, Edinburgh, UK) active (includes both walking and standing behaviors); IQST = IceQube count of the number of steps. 


\section{A) Standardized OBA and ACT variables}

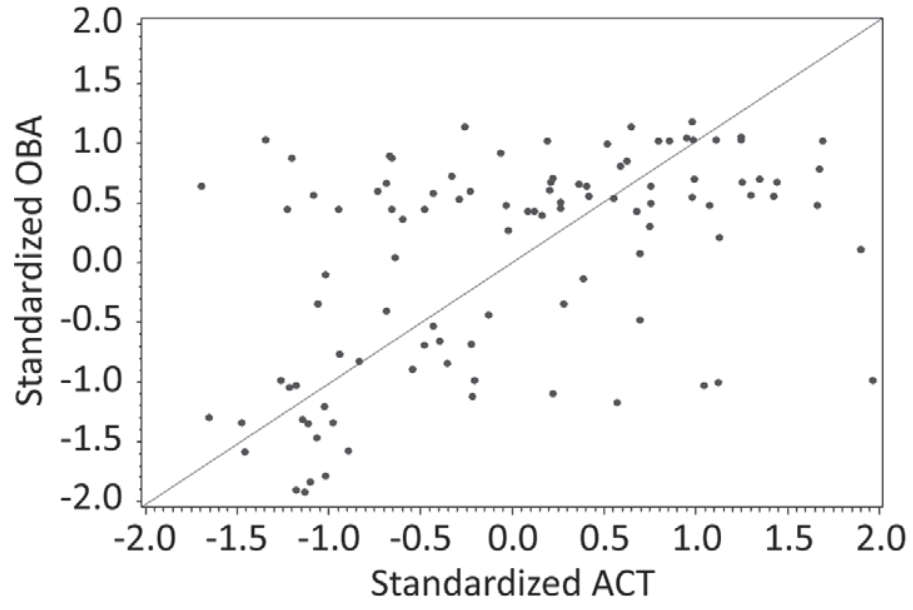

C) Standardized OBW and IQST variables

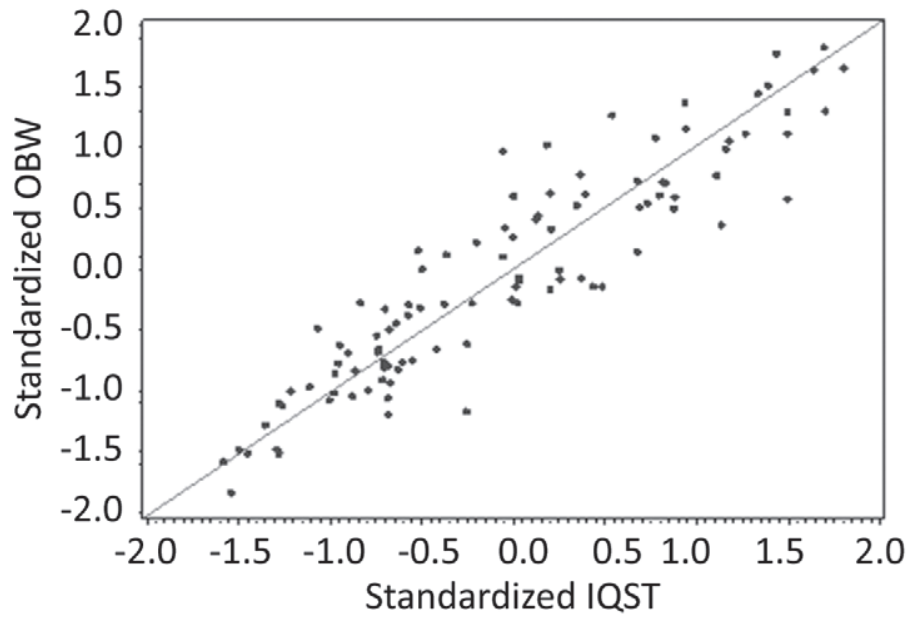

B) Standardized OBW and ACT variables

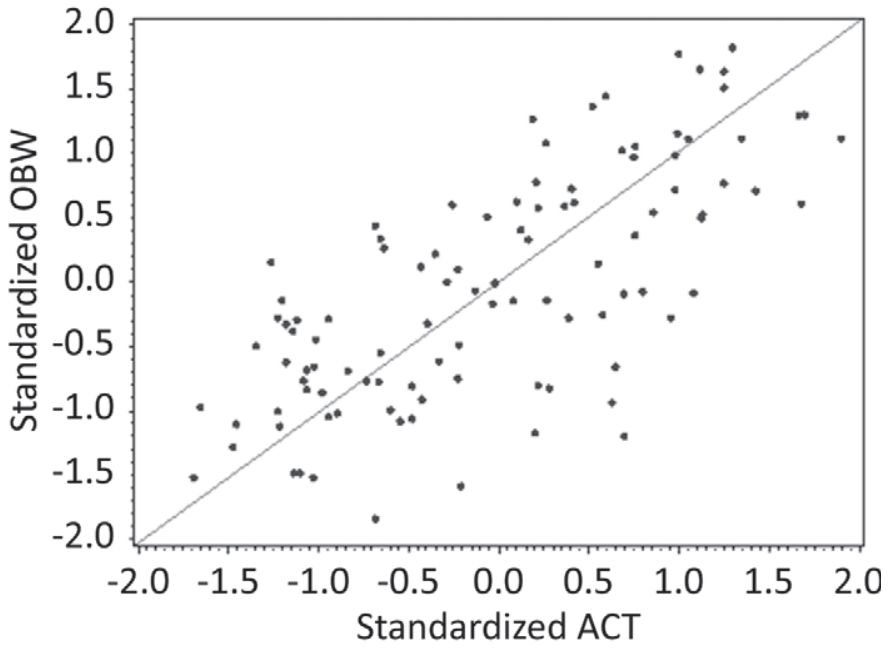

D) Standardized OBW and IQA variables

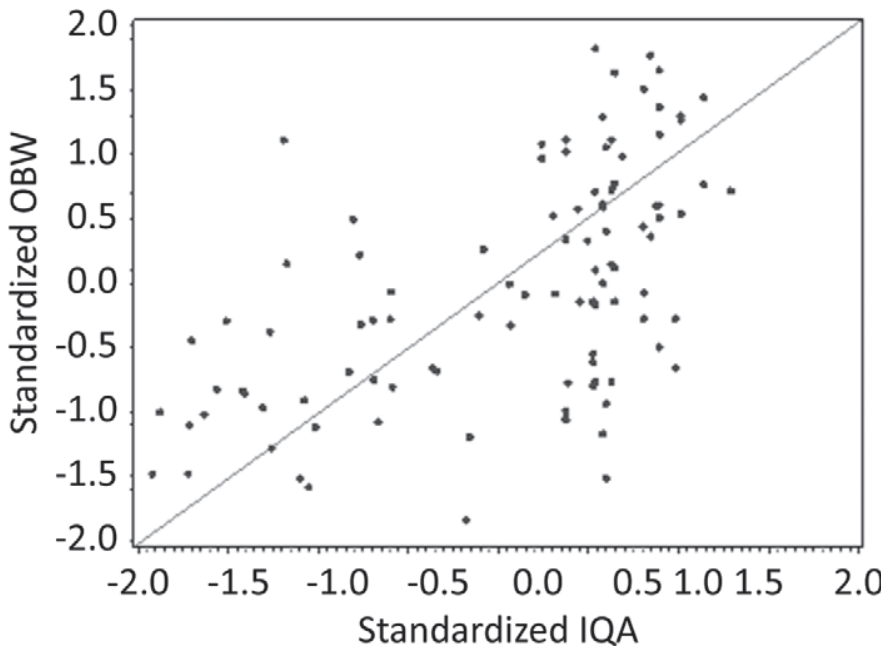

Figure 1. Graphs of standardized (mean $=0$, variance $=1$ ) of $(\mathrm{A})$ live observation active (OBA) with Lely activity unit (ACT; Lely, Maassluis, the Netherlands), (B) live observation walking (OBW) with ACT, (C) live observation walking (OBW) with IceQube pedometer steps (IQST), and (D) OBW with IceQube pedometer (IceRobotics, Edinburgh, UK) activity (IQA).

graphed to show the relationship between RUM and OBRUM (Figure 5).

The Bland-Altman analysis (Figure 6) indicated a lack of agreement between RUM and OBRUM, with a difference of $50 \mathrm{~min}$ or more for some observations. The RUM may record approximately 29 min below or 37 min above the time reported by OBRUM. These values are outside the boundaries determined as acceptable by the $95 \%$ confidence interval. However, it should be considered that the classical Bland-Altman analysis was created for simpler experimental designs and it does not account for the within-subject variability present in our repeated-measures experiment.

\section{DISCUSSION}

The activity units calculated by ACT provided a depiction of walking behavior in dairy cattle, but with lower resolution than other technologies, such as the IQ, or the performance of live observations by humans. The ACT is not as accurate as capturing other postural behaviors, such as lying, which are important for assessing the welfare and comfort of cattle. Optimizing lying time is important to ensure good welfare and production of dairy cattle because reduced lying time has physiological consequences. For example, decreased lying times have been shown to decrease blood plasma 
Table 5. Examination of individual cow rumination behavior using Pearson correlations $\left(\mathrm{r}_{\mathrm{p}}\right.$ ) between rumination monitored by activity monitor (RUM; Lely, Maassluis, the Netherlands) and live observations of rumination (OBRUM)

\begin{tabular}{lccc}
\hline Cow ID & $\begin{array}{c}\text { Observations } \\
\text { (no.) }\end{array}$ & $\mathrm{r}_{\mathrm{p}}$ & $P$-value \\
\hline 2524 & 4 & 0.66 & 0.337 \\
2543 & 5 & 0.78 & 0.122 \\
2606 & 4 & 0.99 & 0.010 \\
2613 & 2 & 1.00 & - \\
2616 & 7 & 0.79 & 0.036 \\
2627 & 4 & 0.99 & 0.008 \\
2717 & 4 & -0.78 & 0.223 \\
2722 & 3 & 0.99 & 0.023 \\
2723 & 5 & -0.06 & 0.929 \\
2734 & 5 & 0.24 & 0.703 \\
2737 & 4 & 0.98 & 0.022 \\
2808 & 5 & 0.77 & 0.131 \\
2818 & 4 & 0.87 & 0.127 \\
2822 & 5 & 0.83 & 0.085 \\
2841 & 2 & 1.00 & - \\
\hline
\end{tabular}

concentrations of growth hormone, which may affect milk production and indicate the homeostatic cost of a challenging physical environments (Munksgaard and Løvendahl, 1993).

The ACT correlation to standing idle was moderate $\left(r_{p}=0.46\right)$, with $22 \%$ of the OBS variance accounted for by the ACT, which is smaller than that of other relationships (e.g., IQA with OBS, $\mathrm{r}_{\mathrm{p}}=0.94$ with $88 \%$ variance). If a cow is inactive because she is standing idle for a long period, this behavioral pattern may indicate that the lying surface is uncomfortable (Leonard et al., 1994) or that the cow is unable to find a stall to lie down in as a result of overcrowding (Haley et al., 2000).

The cows in this study would stand while grazing or, on very warm days, stand in the barn under a large fan. Automated information about changes in walking and lying behavior can provide insight into cow health and reproduction. When an individual cow is ready to be bred, there will likely be an increase in walking and mounting behavior, and therefore in ACT activity units, when a cow is entering estrus (Hurnik et al., 1975). Alternatively, decreased walking, and therefore decreased ACT activity values, could indicate that a cow is ill or injured and requires further assessment and possible treatment (Edwards and Tozer, 2004).

The IQ data correlated very well with human observation, accounting for 81 to $94 \%$ of the variance of the gold standard of live observations. In turn, ACT correlated very well with IQST, and was also consistent with a high correlation with some live observation measures, accounting for $37 \%$ of the variance of human observation for walking. Further, ACT data correlated, to the same degree, with IQ and OB data for activity and for walking.

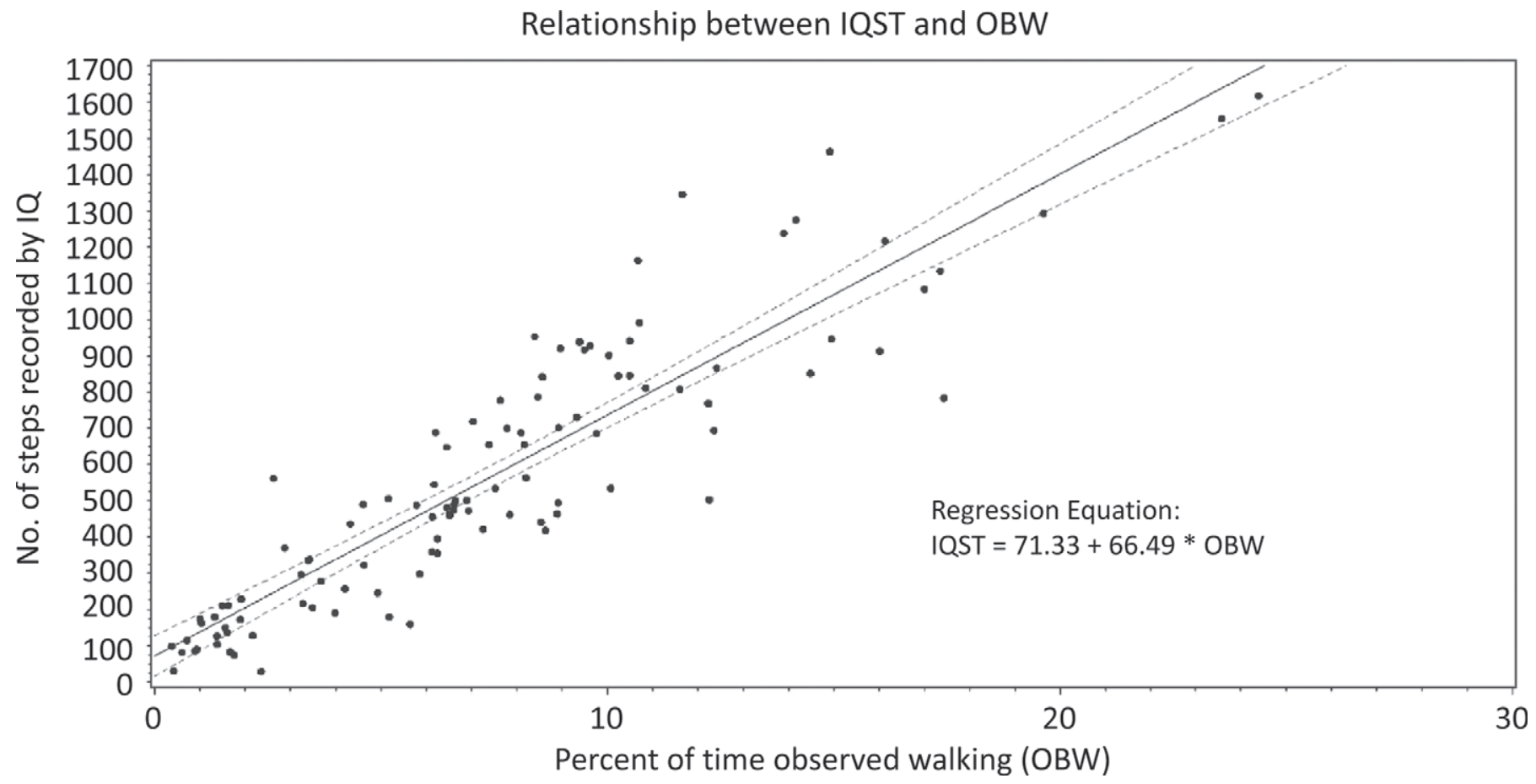

Figure 2. Live observations of walking compared with the number of steps taken by the cow as recorded by IceQube (IQ) pedometers (IceRobotics, Edinburgh, UK). The solid line is the regression line of the data and the dashed lines are the $95 \%$ confidence limits. 


\section{Relationship between ACT and IQST}

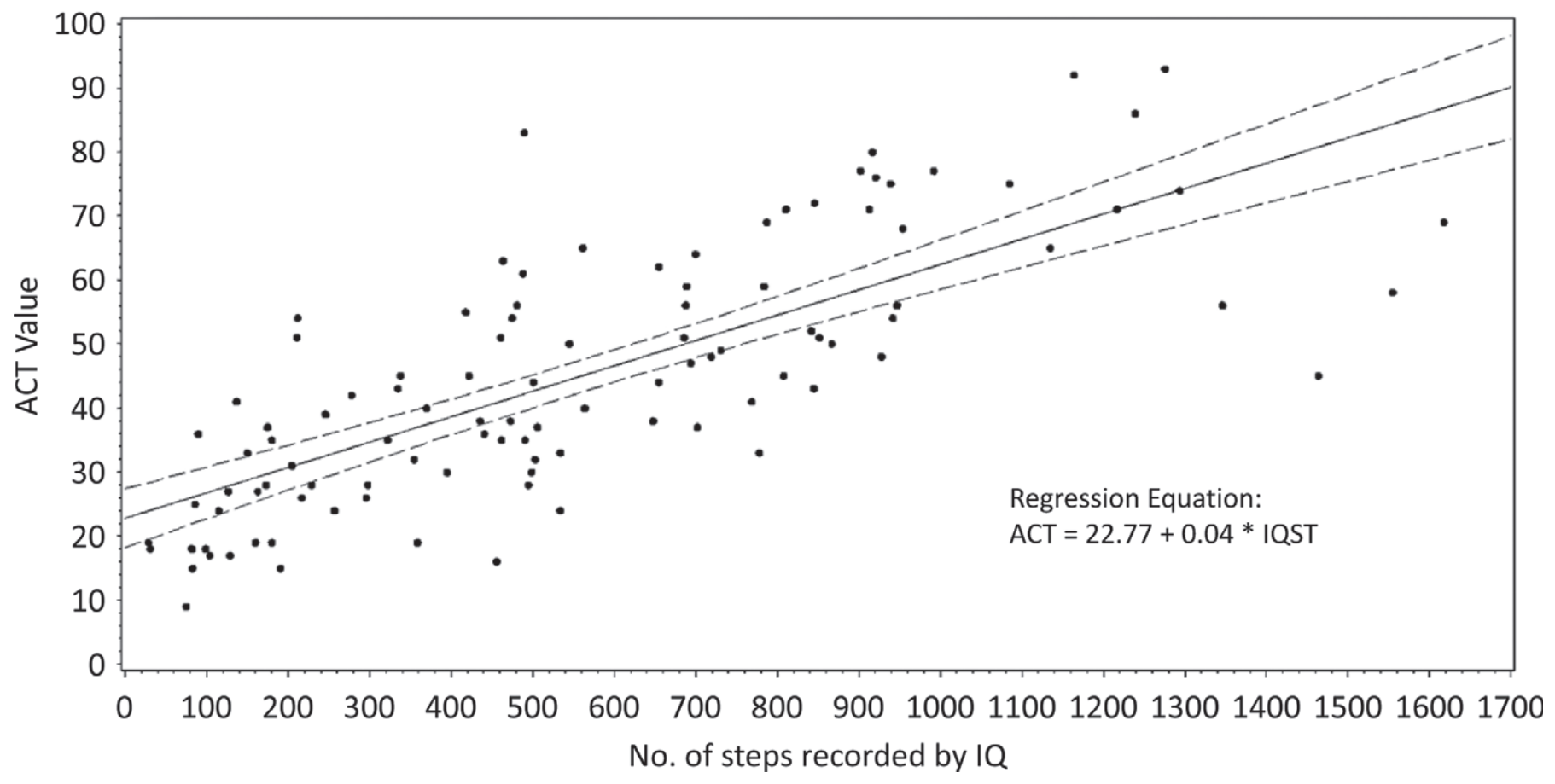

Figure 3. Data obtained from automatic milking system (AMS) activity monitors (Lely, Maassluis, the Netherlands) compared with measurement of the number of steps taken by a cow obtained from IceQube (IQ) pedometers (IceRobotics, Edinburgh, UK). The solid line is the regression line of the data and the dashed lines are the $95 \%$ confidence limits.

\section{Relationship between ACT and OBW}

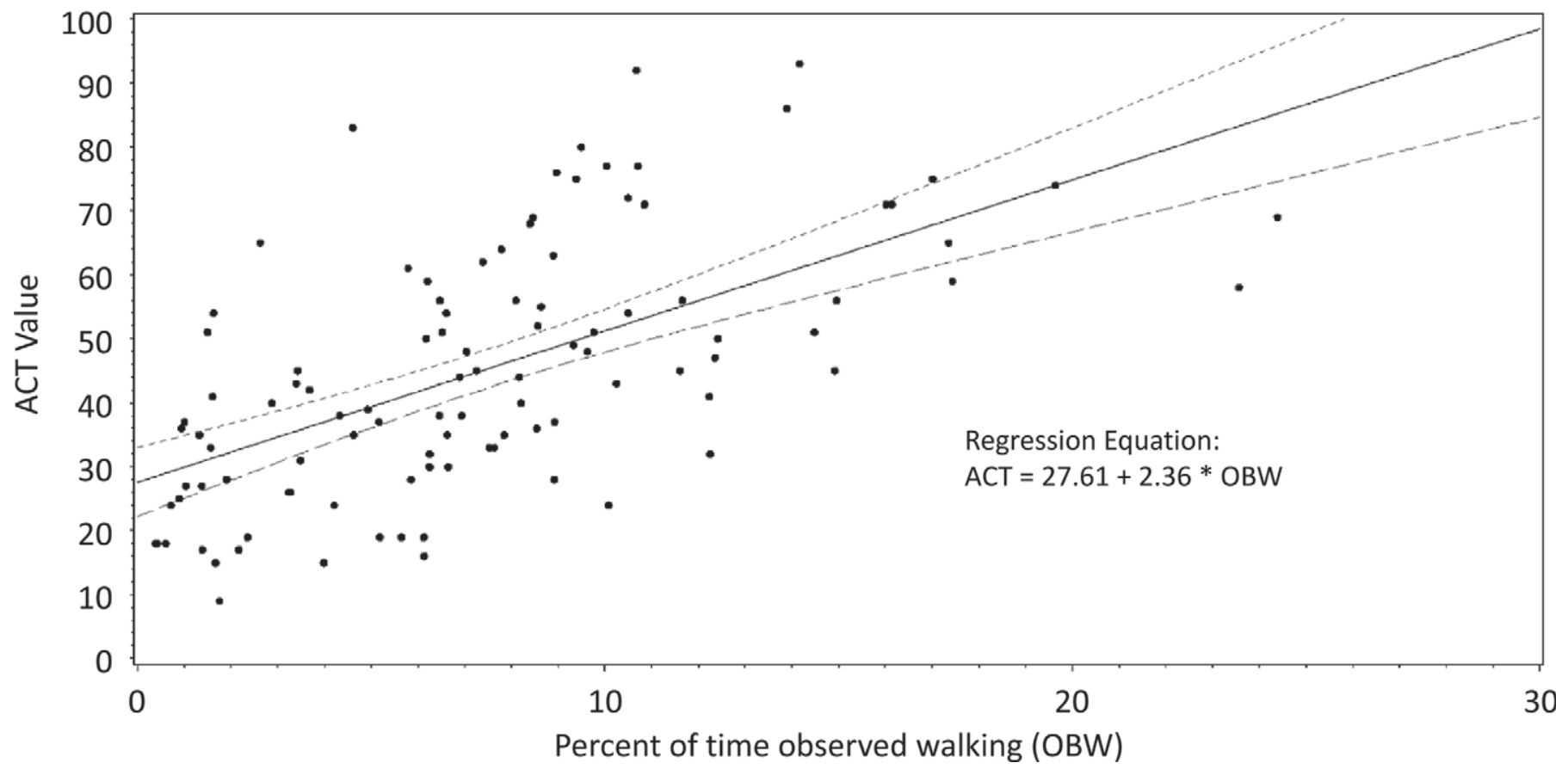

Figure 4. Data obtained from automatic milking system (AMS) activity monitors (Lely, Maassluis, the Netherlands) compared with data obtained from live observations of cows walking. The solid line is the regression line of the data and the dashed lines are the $95 \%$ confidence limits. 
Time spent ruminating

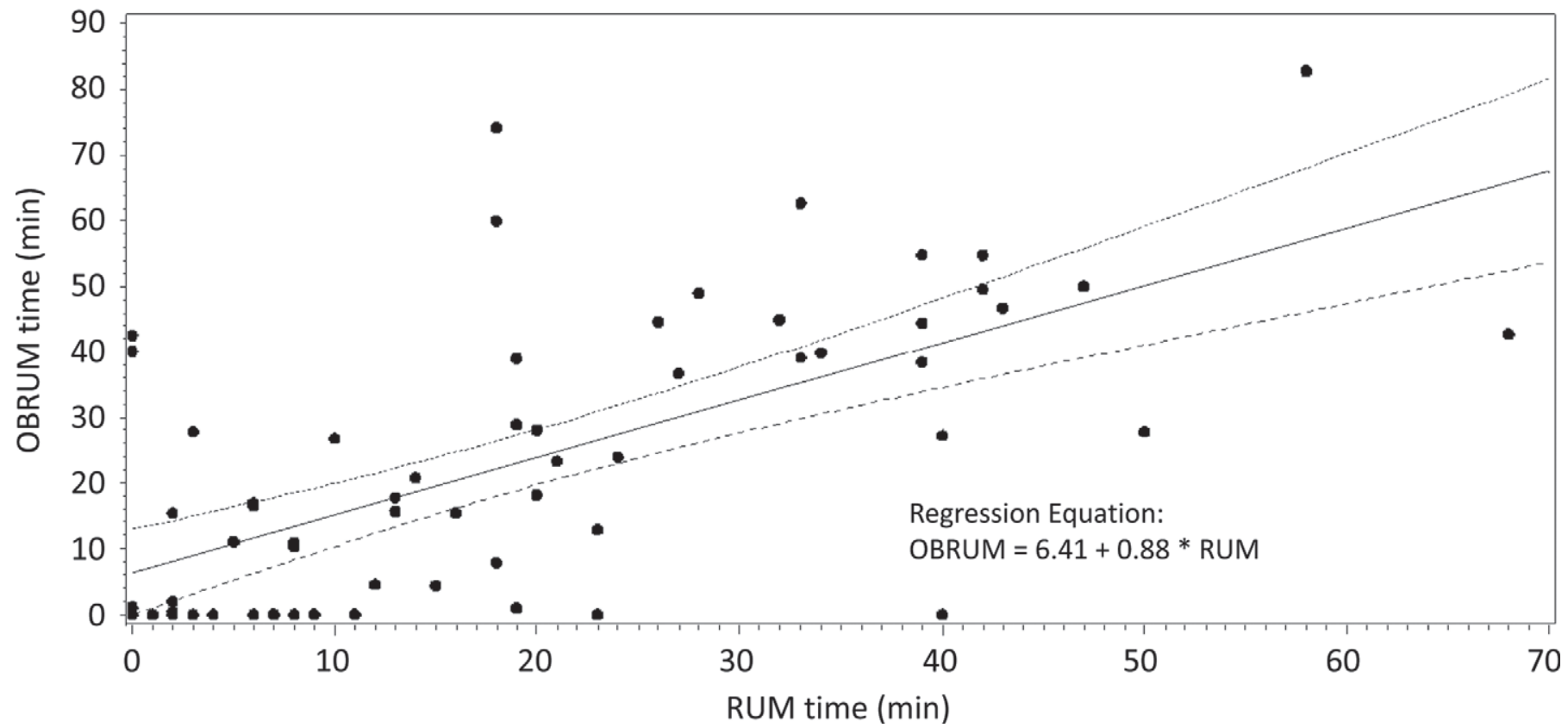

Figure 5. Relationship of time spent ruminating (RUM time) as recorded by automatic milking system (AMS) rumination monitors (Lely, Maassluis, the Netherlands) compared with data collected via live observation (OBRUM time). The solid line is the regression line of the data and the dashed lines are the $95 \%$ confidence limits.

The correlation of RUM with OBRUM was significant, but much lower than what would be acceptable if it were an interobserver reliability estimate between human observers (85\% or greater). Multiple reasons might explain why the correlation between live and monitor measures was lower than expected. First, this correlation may be somewhat low because of difficulty in observing rumination, whether as a result of grass height, distance of cows from observers on pasture, and position of cow's heads away from observers. As a result of observation periods occurring when cows' heads were not visible, fewer data points were recorded by OBRUM for comparison with RUM.

Although we assumed all monitors were functioning normally at the start of the experiment, possible malfunctions of the rumination microphone, improper placement of the sensor on the cow's neck, or environmental interference could have influenced data collection. The rumination microphone is a separate component of the AMS transponder and anecdotally seems to be the part of the device that is first to malfunction (Howard Straub III, Kellogg Biological Station, Hickory Corners, MI, personal communication). If the collar is not placed correctly on the cow's neck (i.e., too high, low, loose, or tight), this may also interfere with sounds of rumination being recorded by the monitor. Thus, it is important to ensure proper placement of the collar on each animal to minimize failure due to human error. It was suspected that equipment error was responsible for the low or negative correlations between OBRUM and RUM for 3 of the cows on this study.

Future work to better validate the rumination monitor would include observing rumination behavior in a setting where the cow's head is always within view of the observer, such as in a tiestall barn. Tests on the proper placement and tightness of the collars on the cows' necks would provide producers and researchers with information on exactly where the collar should be placed and how much accuracy can be expected in the outputs when placement deviates from this physical location.

Understanding exactly how automated body-worn monitors record behavioral data and what data they record are valuable for researchers because outputs from such devices could be used to create a comprehensive data set (i.e., $24 \mathrm{~h}$ including dark periods or over long periods of time) or to gather information that is otherwise very time consuming or expensive to collect. This was the first study to validate the Lely ACT and RUM on a pastured-AMS dairy. The data obtained from ACT does provide insight into the walking behavior of dairy cows that is easily accessed via the management program, is available immediately, and does not require intensive human labor to directly observe the cows. This 


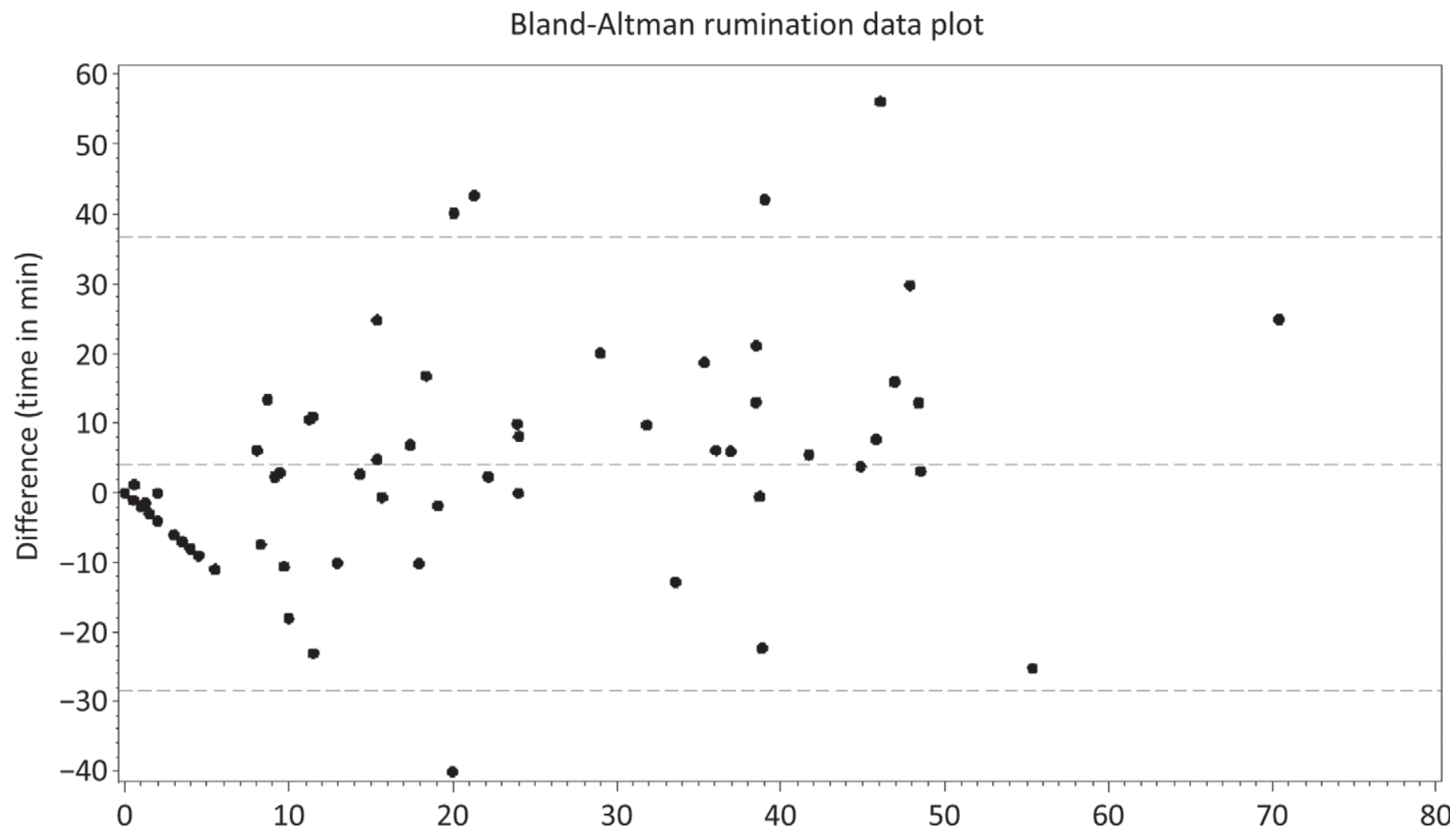

Figure 6. A Bland-Altman plot depicting rumination data (time in min from all 15 cows). The upper horizontal line in the plot corresponds to the higher $95 \%$ confidence limit (36.59 min). The central horizontal line corresponds to the mean of the difference between methods (OBRUM $-\mathrm{RUM}=4.01 \mathrm{~min})$, and the lower horizontal line corresponds to the lower $95 \%$ confidence limit $(-28.56 \mathrm{~min}) . \mathrm{OBRUM}=$ live observations of rumination; RUM = data from automatic milking system rumination monitor (Lely, Maassluis, the Netherlands).

is especially important in an extensive pasture-based system, where it may be challenging to locate specific cows for direct visual observation; the output from the ACT and RUM will provide a manager or herdsman information on these important maintenance behaviors. Although the ACT correlation was not as high as that of IQ for recording walking behavior, it is valuable tool that producers may find helpful in determining breeding periods for cows or in early detection of illness or injury. Additionally, the ACT does not require additional devices to be placed on the cow or increased labor in downloading and interpreting the results, as is necessary with the IQ and other similar technologies. Producers can benefit from the information collected by monitors to gain more precise individual behavioral data across time and compared with conspecifics. After habituation, remote body-worn monitors do not alter cow behavior (MacKay et al., 2012), providing a more accurate picture of what the cow is doing than information that can be collected by human observation (due to the observer effect) or video recording (due the limited field of view of cameras).
Beyond cow comfort, individual data can provide insight on the individual's health status. Subtle shifts in cow activity or rumination are important to detect subclinical diseases or injuries before they progress into a major health concern for a cow. For example, metritis can be predicted by changes in feeding and social behavior 2 wk before an individual cow shows clinical signs of infection (Huzzey et al., 2007).

Monitors associated with AMS have an advantage with regard to data presentation and collection compared with the IQ. While the cow is in the milking herd, ACT and RUM are constantly collecting and reporting information to the AMS, providing results virtually in real time. The collars do not need to be removed for data to be downloaded or to have the batteries changed, and information is reported to the computer system multiple times throughout the day. The AMS monitors are fully integrated management tools that work within a unified system so only one set of software has to be learned and utilized. Rumination and activity data can be examined alongside the cow's milk production, the number of times she has 
visited the AMS, milk quality parameters that might signal mastitis, BW, and how much concentrate she has eaten to provide the producer a powerful management tool to understand and monitor each individual cow in the herd with low labor inputs. The application of $\mathrm{ACT}$ and RUM for research purposes would be more limited because other devices exist that provide more accurate and detailed information. Although ACT and RUM may be useful to provide additional or adequate information, depending on exactly what research questions are being asked, these monitors are limited to reporting information about general activity levels and rumination, whereas the IQ monitor provides a much more accurate depiction of all locomotion and postures.

\section{CONCLUSIONS}

The ACT was accurate at reporting how much a cow moved; however, it provided no information about lying or standing behaviors, which should be quantified to have a comprehensive understanding of an individual cow's health and comfort in the environment. The RUM provided information on the amount of time an individual spent ruminating during the day; however, further research is needed to ensure the monitor is functioning properly, is placed correctly, and has minimal interference from the environment before this piece of technology can be fully validated.

\section{ACKNOWLEDGMENTS}

Support for this study was received from a grant to Michigan State University from the W. K. Kellogg Foundation (Battle Creek, MI) to establish a pasturebased dairy program at the Kellogg Biological Station (KBS; Hickory Corners, MI). The authors extend sincere thanks to all those who assisted with this research and manuscript preparation. This project would have not been possible with the assistance of Rob Ashley and the farm staff at KBS, Santiago Utsumi and Kate Steensma at KBS, and Jacquelyn Jacobs (University of Guelph, Guelph, ON, Canada), and Denae Baker and Julie Chamness (both of Michigan State University, East Lansing, MI) for their assistance with data collection in the field. Assistance with the statistical analysis was provided by Robert J. Templeman, and the College of Agriculture and Natural Resources Statistical Consulting Center (Michigan State University, East Lansing), with additional support by Rick D'Eath (Scotland's Rural University College, Easter Bush, Midlothian, UK). Comments and suggestions from
Peter Krawczel (University of Tennessee, Knoxville) were very helpful in refining the manuscript.

\section{REFERENCES}

Edwards, J. L., and P. R. Tozer. 2004. Using activity and milk yield as predictors of fresh cow disorders. J. Dairy Sci. 87:524-531. http://dx.doi.org/10.3168/jds.S0022-0302(04)73192-6.

Haley, D. S., J. Ruschen, and A. M. de Passillé. 2000. Behavioral indicators of cow comfort: Activity and resting behavior of dairy cows in two types of housing. Can. J. Anim. Sci. 80:257-263. http:// dx.doi.org/10.4141/A99-084.

Hurnik, J. F., G. J. King, and H. A. Robertson. 1975. Estrous and related behaviour in postpartum Holstein cows. Appl. Anim. Ethol. 2:55-68. http://dx.doi.org/10.1016/0304-3762(75)90065-6.

Huzzey, J. M., D. M. Veira, D. M. Weary, and M. A. G. von Keyserlingk. 2007. Prepartum behavior and dry matter intake identify dairy cows at risk for metritis. J. Dairy Sci. 90:3220-3233. http:// dx.doi.org/10.3168/jds.2006-807.

LeBlanc, S. 2010. Monitoring metabolic health of dairy cattle in the transition period. J. Reprod. Dev. 56 (Suppl.):S29-S35. http:// dx.doi.org/10.1262/jrd.1056S29.

Ledgerwood, D. N., C. Winckler, and C. B. Tucker. 2010. Evaluation of data loggers, sampling intervals, and editing techniques for measuring the lying behavior of dairy cattle. J. Dairy Sci. 93:5129-5139. http://dx.doi.org/10.3168/jds.2009-2945.

Leonard, F. C., J. O'Connell, and K. O'Farrell. 1994. Effect of different housing conditions on behavior and foot lesions in Friesian heifers. Vet. Rec. 134:490-494. http://dx.doi.org/10.1136/vr.134.19.490.

MacKay, J. R. D., J. M. Deag, and M. J. Haskell. 2012. Establishing the extent of behavioural reactions in dairy cattle to a leg mounted activity monitor. Appl. Anim. Behav. Sci. http://dx.doi. org/10.1016/j.applanim.2012.03.008.

Martiskainen, P., M. Järvinen, J. P. Skön, J. Tiirikainen, M. Kolehmainen, and J. Mononen. 2009. Cow behavior patter recognition using a three-dimensional accelerometer and support vector machines. Appl. Anim. Behav. Sci. 119:32-38. http://dx.doi. org/10.1016/j.applanim.2009.03.005.

McGowan, J. E., C. R. Burke, and J. G. Jago. 2007. Validation of a technology for objectively measuring behavior in dairy cows and its application for oestrous detection. Proc. N.Z. Soc. Anim. Prod. 67:136-142.

Müller, R., and L. Schrader. 2003. A new method to measure behavioural activity levels in dairy cows. Appl. Anim. Behav. Sci. 83:247-258. http://dx.doi.org/10.1016/S01681591(03)001412.

Munksgaard, L., and P. Løvendahl. 1993. Effects of social and physical stressors on growth hormone levels in dairy cows. Can. J. Anim. Sci. 73:847-853. http://dx.doi.org/10.4141/cjas93-087.

O'Driscoll, K., L. Boyle, and A. Hanlon. 2008. A brief note on the validation of a system for recording lying behavior in dairy cows. Appl. Anim. Behav. Sci. 111:195-200. http://dx.doi.org/10.1016/j. applanim.2007.05.014.

Ruiz-Garcia, L., L. Lunadei, P. Barreiro, and J. I. Robla. 2009. A review of wireless sensor technologies and applications in agriculture and food industry: State of the art and current trends. Sensors (Basel) 9:4728-4750. http://dx.doi.org/10.3390/s90604728.

Urton, G., M. A. G. von Keyserlingk, and D. M. Weary. 2005. Feeding behavior identifies dairy cows at risk for metritis. J. Dairy Sci. 88:2843-2849. http://dx.doi.org/10.3168/jds.S00220302(05)72965-9.

Watanabe, N., S. Sakanoue, K. Kawamura, and T. Kozakai. 2008. Development of an automatic classification system for eating, ruminating and resting behavior of cattle using an accelerometer. Grassl. Sci. 54:231-237. http://dx.doi.org/10.1111/j.1744697X.2008.00126.x. 